\title{
Challenges Face during Fitting of RGP Contact Lenses in Keratoconus
}

\section{Shivam Bajoria*}

Department of Paramedical/Ophthalmology, TMU, College of Paramedical

Sciences, India

*Corresponding Author: Shivam Bajoria, Department of Paramedical/

Ophthalmology, TMU, College of Paramedical Sciences, India.
Received: June 01, 2020

Published: September 11, 2020

(C) All rights are reserved by Shivam Bajoria.

\begin{abstract}
Introduction: RGP contact lenses is of paramount importance in Keratoconus patient management to rehabilitate vision and improve patients quality of life.

There's always been on focus to find alternative to Contact lenses options which is already been proposed.

Like if patient show contact lens intolerance or disease progresses, basically the surgical procedures along with the non optical options can be applied, as looking on to which option the patient is more convenient according to his requirement.

Also there's can be an option of Scleral Contact Lenses which can even be the first choice if the RGP intolerance occurs or there can be the option of Kerasoft lenses if the comfort is the main priority of the patient.

Keywords: RGP Contact Lenses; Soft Contact Lenses; Keratoconus; Scleral Contact Lenses; Rose K Contact Lenses; Kerasoft Lenses; PROSE Design; Hybrid Contact Lenses; Piggy Back Contact Lenses; Prose Zones
\end{abstract}

\section{Introduction}

RGP contact lenses is of paramount importance in Keratoconus patient management to rehabilitate vision and improve patients quality of life.

\section{Aim of the Study}

Challenges Face During Fitting of Gas Permeable Contact Lenses in Keratoconus.

\section{Objective of the Study}

The main objective which I think has come through this study is, When the old conventional method do not get suitable to the patient we need to find other methods which may fulfill the need, it can be either surgical or non-surgical.

Taking an example of a particular case of a soccer player keeping in mind of his dynamic work environment and satisfying the need for stable correction and constant fulfilled of view demand without spectacles, the GP contact lenses may or may not satisfy the needs.
We need contact lenses of such design, which is Thick fenestrated design getting compatible with his suitable dynamic environment and all basic needs.

Need of study: The main focus of the study is on discussing about surgical and Non - surgical methods of treatment when old conventional method has proven to be a challenge.

\section{Methodology}

Coming on to the methodology of the study - we should first discuss WHAT IS KERATOCONUS?

So, it's a non inflammation ectatic condition of cornea giving it a conical shape.

It is usually Bilateral $85 \%$.

It is a Multifactorial disease with, Genetic, Biochemical, Biomechanical and pathophysiology.

Where some of its peculiar symptoms are: Progressive blurring of vision, halos around lights, light sensitivity, ocular irritation. 
Visual loss occurs primarily from irregular astigmatism and myopia and secondarily from corneal scarring.

While some of its important signs are:

- Window reflex on cornea becomes distorted.

- $\quad$ Placido disc examination shows irregularity of the circles.

- A yawning reflex on retinoscopy and high oblique or irregular astigmatism is obtained.

- Slit lamp examination shows thinning and ectasia of central cornea, opacity of the apex, Fleischer's ring at the base of cone and folds in Descemet's and bowman's membrane.

Some of the associated ocular complications with keratoconus are:

- $\quad$ Ectopia lentils, congenital cataract, aniridia, retinitis pigmentosa, vernal keratoconjunctivitis (VKC), floppy eyelid syndrome and corneal endothelial dystrophy.

- While acute hydrops may complicate the keratoconus due to rupture of Descemet's membrane. The condition is characterized by sudden development of corneal oedema associated with marked defective vision, pain, photophobia and Lacrimation.

While some of the systemic conditions associated with keratoconus are: Marfan's syndrome, atopy, asthma, eczema, hay fever, Downs syndrome, Ehlers - Danlos syndrome, osteogenesis imperfecta and mitral valve prolapse.

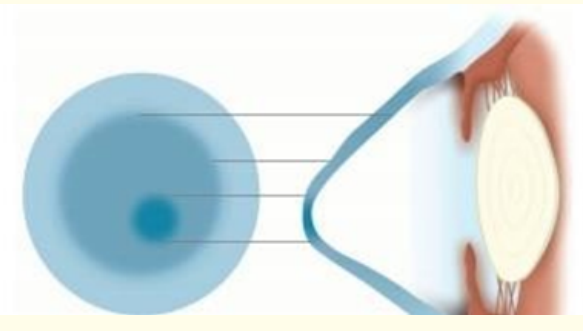

Figure 1: Diagrammatic depiction of cone shaped cornea.

\section{Examination}

As fitting GP contact lenses in the case of Keratoconus is a proven convenient method and it's diagnosis and management is itself a big challenge.

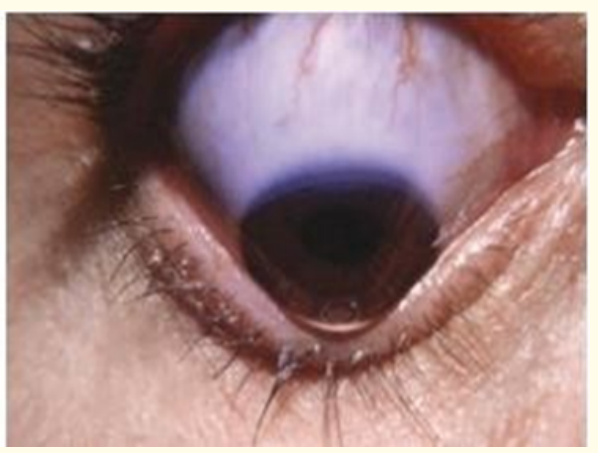

Figure 2: Clinical Photograph Munson's sign.

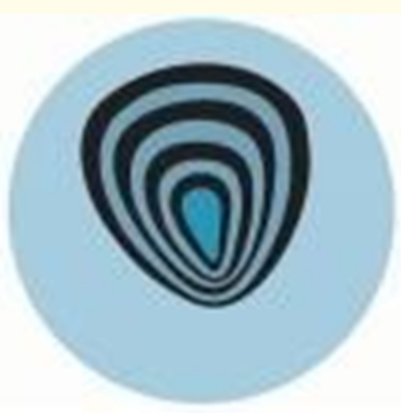

Figure 3: Irregular circles on Placido Disc.

Keeping in mind three challenges

- First difficult is link to an accurate identification of Keratoconus patient clear gradation and diagnosis needs to be done.

- The second challenge is link to distance classification because there is no clinically adequate classification system for Keratoconus disease.

- Whereas, Third challenge could be it's gradation and treatment.

Non - surgical approach may be the first action in patient.

Keeping in mind the suitable surgical procedures like: Corneal Cross Linking (CXL), have been proposed in patient younger than 40 years to halt disease progression.

Whereas, Descematic deep Anterior Lamellar Keratoplasty are performed in patients with Descemet's membrane compromise or another surgical choice may be Penetrating Keratoplasty. 
Also, the Intra Corneal Stromal Ring Segment ICRS increase the corneal stability, help in normalisation of the corneal contour normally in keratoconus grade 3 .

Recently, a new clinically validated open access web calculator (calculens.com) has been developed with the aim to aid contact lenses practitioner of the GP Lens to be fitted in Keratoconus patient.

This new tool will allow that Keratoconus patient receive the most adequate lens and help the practitioner to provide a second fitting process decrease the number of diagnosis lenses trials and chair time to those achieved in Standard GP Contact lenses fitting.

Now we need to discuss on Newer Contact Lenses Method for the treatment which has come out to be; Use of soft contact lenses.

As on the other hand conventional Non - optical correction of GP contact lenses may not be suitable under various circumstances for that Soft $\mathrm{K}$ is a new soft lens compromising a thick fenestrated design to fit in with mild to moderate corneal distortion and fitting problems or physical intolerance to RGP contact lenses.

Study site: Going to Be Decided On the basis of My Internship Venue Further This Year.

\section{Case Report}

A case report has been discussed which is basically based upon: How to Get Best Fit for Keratoconus!

A 17-Year-old male patient has come to an O.P.D of an Ophthalmological Department complaining of gradual painless decrease in vision in both eyes since 6-7 years.

Using glasses for 6 years and PGP is been noted 4 months old.

He was diagnosed with OU keratoconus.

RGP contact lenses were applied but they were not comfortable.

His family and allergy history was nil.

- $\quad$ Distance Visual acuity (unaided) - OD - 20/400 -----20/60, OS - 20/400 ----20/60.

- $\quad$ Distance visual acuity with glasses - OD - 20/60, OS - 20/50.

- Near Visual acuity - OD - N6@40cms, OS - N6 @ 40cms.

- Present Glass prescription - OD: -6.75Dsph/-1.75Dcyl*100, OS: -6.50 Dsph/-2.50Dcyl*80.
- Objective refraction (Scissors reflex): OD: -8.00Dsph/3.00Dcyl*100, OS: -7.00Dsph/-3.50Dcyl*180.

- Subjective refraction - OD: -7.00DS/-3.50Dcyl*100, OS: -7.00DS/-2.50Dcyl*80.

- Subjective refraction visual acuity: OD - 20/50, N6@40 cms, OS - 20/50, N6@40 cms.

- $\quad$ Slit lamp examination - Eye lids: OU flat.

- $\quad$ Conjunctiva - OU Quiet.

- $\quad$ Sclera - OU Normal.

- Cornea - OU as shown.

- $\quad$ A.C - OU Normal in contents and depth.

- Iris - OU Normal pattern and color.

- $\quad$ Pupil - OU R/R/R.

- $\quad$ Crystalline lens - OU clear

- $\quad$ IOP - OU Digitally normal.

Under the slit lamp cornea is associated with Fleischer's ring and apical scarring.

Now Corneal topography had been performed which shows four maps in it as follows:

- $\quad$ Anterior surface of cornea.

- Posterior surface of cornea.

- $\quad$ Keratometric value graph.

Corneal thickness map also it is use to measure severity of cone. Where Maximum keratometric value can go up to $60 \mathrm{D}$.

Now, based upon Krumeisch classification the keratoconus has been found to be of Grade IV as:

- $\quad$ Keratometry value is above 55D.

- $\quad$ Thickness is above 200 .

- $\quad$ Spherical Equivalent is not measured. 
- And corneal scars can be seen.

- Now, the treatment options can be various;

- It can be Glasses, Contact Lenses, Surgical.

- Among Contact lenses there can be varied options like Soft (Spherical, Toric, Kerasoft)

- $\quad$ RGP and Rose -K.

- $\quad$ Piggy back.

- Hybrid.

- And another option can be Scleral Contact Lenses.

- When comfort is the main priority of the patient we choose Kerasoft lenses as an option.

- Whereas RGP is the most common choice which provide good quality vision but it's slightly may show discomfort to the patient for that adaptive period is suggested for RGP lenses.

- Mainly we use Tricurve design in RGP which is easily available in Indian market

- If RGP is intolerant we may recommend Piggy back lenses.

- Which are a kind of design in which soft lenses are at over side and to the back surface is RGP.

- These lenses provide Good comfort and minimum scarring.

- Whereas, hybrid contact lenses are a combination of rigid and sift lenses.

- And coming on to other option are Scleral contact lenses which can be preferred as the first choice when RGP intolerance occurs.

- Immediately after the refraction the patient was referred to Contact lens clinic where he was advice to wear ROSE - K lenses of multicurve design which provide good alignment over RGP lenses of trifocal design which do not provide a satisfactory alignment.

Corneal Topography in Keratoconus may reveal about the severity of the cone which may be:

- $\quad$ Nipple - cone size -3 mm; diameter chosen: 8.7 - $8.8 \mathrm{~mm}$
- Oval - cone size - $5 \mathrm{~mm}$; diameter chosen: above $9 \mathrm{~mm}$.

- Globus - cone size - 7- $8 \mathrm{~mm}$; diameter chosen - above $9.4 \mathrm{~mm}$.

The cone involve in keratoconic eye of the patient found to be of Globus type.

The fitting philosophies in keratoconus can be of three types: Apical Clearance, Apical Bearing, 3 point touch.

3 Point touch is an acceptable fitting philosophy in Keratoconus as the weight transfer from center to lens gives better stability and a center feathery touch in the periphery provide good relation between Lena and the cornea.

Now, the Rose -K fitting assessment of the patient comes out to be as follows:

- $\quad$ Base curve - OD 6.45, OS - 6.65

- $\quad$ Power - OD: -9.50, OS - 10.50

- $\quad$ Diameter - OD - 9.40, OS - 9.50

- $\quad$ Company - OU Rose - K STD.

Two types of preferred fitting methods are: Static and Dynamic.

In dynamic fitting the lens is well centered, optic zone covers the pupillary area well and movement is optimum.

Whereas in static fitting it doesn't looks like a 3 point touch.

Though it is also acceptable.

Over refraction comes out to be:

- OD: Plano 20/30, N6 with English chart @30 cm RI

- OS: Plano 20/30, N6 with English chart @ 30 cm RI.

The patient was intolerant to ROSE - K lenses as blink discomfort was noted along with watering of eyes.

When the patient was told about available options as: Hybrid or Scleral lenses.

He chose Scleral lenses.

History: As it is based on a initial design of Leonardo da Vinci in $15^{\text {th }}$ century. 
In $18^{\text {th }}$ century the glass for Scleral lens material got introduced. As because of glass hypoxic changes along with discomfort was noted, though it was helpful in correcting irregularity of cornea and provide better vision.

In $19^{\text {th }}$ century PMMA lenses were introduced.

The oxygen permeability was zero from these lenses.

In 1970-80: Gas permeable contacts were introduced where fluorescein was used as an acrylate material in Scleral lenses.

The penetration was good and the health of the cornea was reported good.

In 1994: Penetration Scleral lenses got introduced which gave very successful results over these years.

\section{What is PROSE?}

Prosthetic Replacement of Ocular Surface Ecosystem.

Its diameter may be - $8-12.5 \mathrm{~mm}, 12.5-15.0 \mathrm{~mm}, 15-25 \mathrm{~mm}$, Mini scleral -15 - $18 \mathrm{~mm}$, Large - 18 - $25 \mathrm{~mm}$.

Whereas diameter of 18 - $18.5 \mathrm{~mm}$ can be preferred in keratoconus and in some other ectatic condition also.

It may be of three major types: Corneal, cornea - scleral and Scleral.

The scleral lens design is available in Curve base design.

Whereas RGP is available in Tri curve design which presents central and two peripheral curves.

Scleral lens design - Central Limbal and two peripheral curves.

Prose design has 3 functional zones:

- Optic - It encloses the fluid reservoir.

- Transitional - It is the center thickness of fluid reservoir.

- Haptic - It rest on the sclera.

Why PROSE Zones?

- $\quad$ Optic zones - It presents a Liquid bandage, it marks the irregularity of cornea, helps in healing if few symptoms, it provide good vision for ocular surface condition also it protects the eyes.
- Transitional zones - It is quite customised in India.

- Haptic zones - It include anti suction safety suction valves that facilitates interchange of tears avoiding passage of air bubbles.

Shape is customised to avoid compression of channels.

It is also use in lofted surface condition also.

What are Micro Channels: Fluid transfer from sclera to tear film.

It gets tear film divided into post and pre Lens Tear Film with micro channels and haptic area which helps to have very good fluid transport available in PROSE design.

What Is Vault Control Technology?

It is gap between lens and cornea.

Saggital height and corneal clearance of lens is dependent on vault.

Vault can be change Independently of the base curve. It can be independently change i.e. increase or decrease the vault.

- $\quad$ Parameter Selection: Diameter.

- $\quad$ Sag height.

- $\quad$ Landing zone fit (Haptic measurement).

- Diameter - Bigger size will provide better alignment and lesser complication.

- It depends on how much space we have in bulbar area.

- We prefer 18 - $18.5 \mathrm{~mm}$ diameter.

- $\quad$ Saggital Height - More sag height provides more steepness.

- $\quad$ Preferable is: 5 - $5.5 \mathrm{~mm}$

- Whereas Flatter cornea leads to lease vault which is preferable $4-4.2 \mathrm{~mm}$

- Haptic Measurement - 15 mm is a preferable haptic Measurement if the lens is on steeper side.

- If the lens is flatter prefer steepening of haptic to be $13 \mathrm{~mm}$.

PROSE fitting evaluation

- Vault: OD: $5.50 \mathrm{~mm}$ 
- OS: $5.50 \mathrm{~mm}$

- Diameter: OD: $18.50 \mathrm{~mm}$

- $\quad$ OS: $18.50 \mathrm{~mm}$

- $\quad$ Base curve: OD: $7.92 \mathrm{~mm}$

- $\quad$ OS: $7.89 \mathrm{~mm}$

- Power: OD: -5.12 D

- OS: $-4.75 \mathrm{D}$

- $\quad$ Haptic (Inner/Outer): OU - 13.75/13.75.

- Vision with PROSE lens: OD: 20/100 ---20/30.

- $\quad$ OS: $20 / 160---20 / 30$.

- $\quad$ Over acceptance: OD: -2.00DS, 20/30 N6

- $\quad$ OS: - 3.00 DS, 20/30, N6

- Value of vault in microns - $300-350$

- Limbal vault in microns - 200

Visual acuity with PROSE lenses:

- $\quad$ OD: $20 / 30, \mathrm{~N} 6$

- $\quad$ OS: 20/30, N6.

Haptic assessment - It provides good alignment and blood flow it should be 360 degrees align on sclera.

It should not have any blanching.

Alignment as far as possible should be good so as the blood flow.

As few of the haptic assessment conditions are given in the image below we will prefer a second case as it fulfils all the parameters of an ideal haptic assessment as discussed above.

Now, when the patient came for a follow up visit after 2 years.

He came with c/o Redness appearance and discomfort after wearing lens for few hours in OU.

H/o wearing lenses 14 hrs a day occasionally sleeping also.

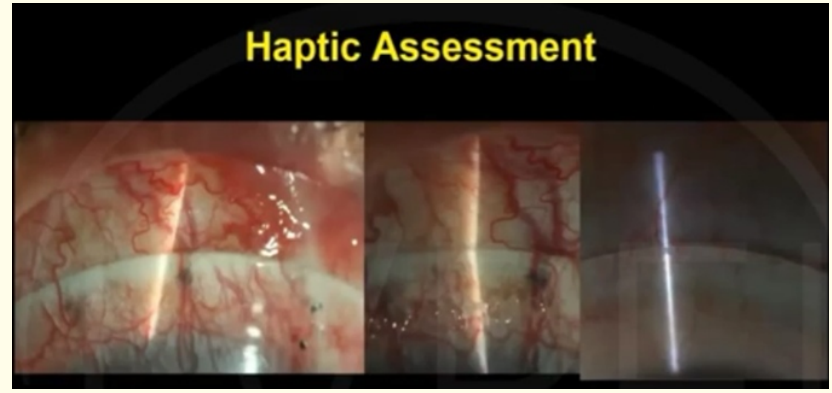

Figure 4

Visual acuity with PROSE lenses:

- $\quad$ OD - 20/30, N6

- $\quad$ OS - 20/25, N6.

Whereas, congestion on bulbar area can be clearly seen.

Fitting assessment found to be a Tight fit and conjunctival indentation can be seen.

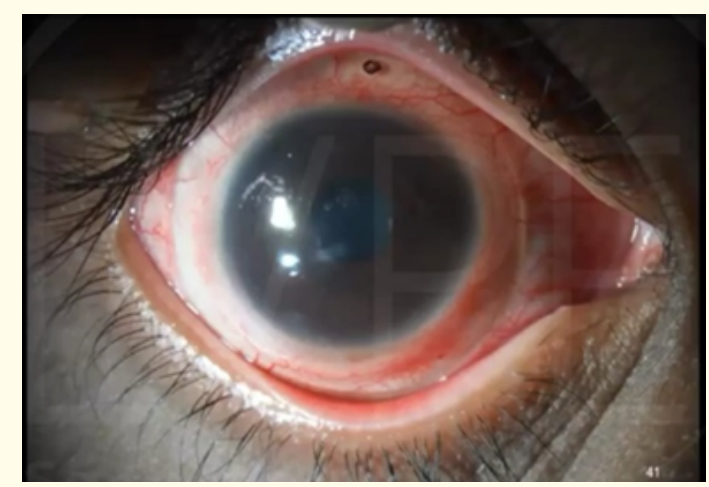

Figure 5

Lens edges are tighter which can be seen clearly in the image, it was congested which shows more congestion on the bulbar area.

Embrigement, Hyperaemia can be seen.

Along with congestion on bulbar and conjunctival area can be seen.

Now, As the patient was wearing the contacts 14 hours a day it has lead to corneal vascularization. 
Corneal vascularization pathogenesis is as follows:

- Hypoxia.

- Stromal hydration and infiltered cells.

- $\quad$ Release of VEGF.

- Neovascularisation.

Therefore, diagnosis of corneal vascularization cells occur which are secondary to hypoxia

- Advice given to the patient at the O.P.D was: To discontinue lenses for 2 weeks.

- Lubricants were prescribed.

- $\quad$ Advice to try High DK material in next visit.

- Then review after 2 weeks with PROSE clinic.

High DK materials are basically available of two types:

- $\quad$ Equalens: Dk - 85.

- $\quad$ Boston X02: Dk: 141.

While in between only the material of the PROSE lens got change.

On the post follow up after 2 weeks:

- While the fitting was acceptable in both eyes.

- No active vascularization was seen.

- Advice of reducing wearing time to 4 hours and after that a gap of few hours was given.

As we can see it clearly in the image below.

Some of the indications of PROSE lenses can be: Therapeutic as well as Optical.

Optical can be as in post surgical condition and few others.

While it can also be indicated in other ectatic conditions like: Dry eyes, Stevens Johnson syndrome.

These are the indications which are other than optical.

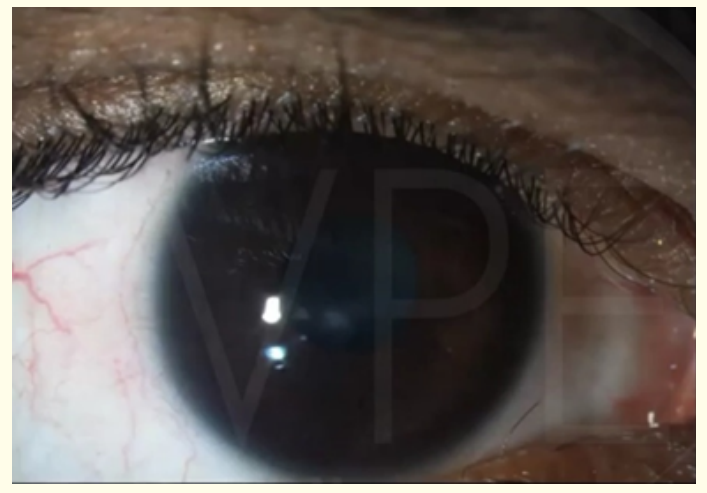

Figure 6

\section{Discussion}

As we have seen in the earlier case reports that the old conventional methods are now getting replaced and newer methods are coming on to apply in the treatment of keratoconus [1-8].

These may be optical, contact lenses as well as Surgical.

The contact lenses may be of various types like:

- $\quad$ Soft lenses, RGP, Scleral, Hybrid, Piggy back contact lenses.

- Whereas the surgical interventions aim at the corneal transplantation of the patient.

- $\quad$ Keeping in mind the diameters, assessment techniques, haptic assessment, vault parameters and few more parameters to be noted we can practice scleral lenses very well in the cases of few ectatic conditions of eye.

Type of study

The type of study is mainly centralised towards, Ideas, editorials, opinion and case report as it is based on a series of patients with an interest.

- Study design: As based on this study;

- Three patients with mild Keratoconus from two 25 year old patients were fitted soft contact lenses.

Both patients had previously worn other contact lens types.

Improvement in comfort and quality of vision compared with previously worn RGP and Soft. 
Toric Contact lens types were the most remarkable advantages objectively observed with subjective described by both patients.

Whereas, one patient is a professional soccer player as discussed earlier the benefits were also important in terms of compatibility with dynamic environment of his activity.

Other than this in another case report we find how the scleral lenses of PROSE design have been in preference over the ROSE - K lenses in the patient of severe keratoconus.

Using lenses of diameter $9.4-9.5$.

\section{Conclusion}

This new soft lens design for irregular corneas is especially Indicated for those with intolerance to RGP for patients for whom RGP Lenses do not satisfy the Dynamic environment inherent to some professional or leisure activity particular sports activity.

This has proven to be a good option to consider in patients with mild to moderate Keratoconus and good correction of slight or moderate irregular corneal astigmatic with good intolerance.

Also the PROSE design Scleral contact lenses can be a very good option in the case of RGP intolerance keeping in mind all the parameters like, dimensions of contact lenses, fitting assessment as discussed earlier and going with the corneal topography reports which depicts about the severity of the keratoconus in a case report, the emphasis on using PROSE design Scleral contact lenses is done over the ROSE - $\mathrm{K}$ of larger diameter close to 9.4 or greater.

Results: The results which comes out by this study is that the Soft lenses and the Scleral lenses of PROSE design are now being use over old conventional RGP lenses as of the patient is RGP intolerant or the disease progression occurs.

Keeping in mind the various parameters such as fitting assessment, diameters and few more we can practice these lenses very well in the case of ectatic conditions of the eye as in keratoconus as discussed.

By the case report we also come to a conclusion that High DK value is a very important parameter in practicing scleral lenses as discussed, as it may increase the oxygen permeability and decrease the congestion, hyperaemia and corneal neovascularization.

Study population: This has come out to be as Three patients have been consider of 25 year old with varied professions.

And a 17 year old male patient.
Study criteria: The criteria is based upon newer surgical and Non surgical methods for the correction along with discussing about few challenges faced in managing a keratoconic patient.

Inclusion criteria: Some of the main inclusion criteria which are the key features for the subject to be included in the study can be persistence mild to moderate keratoconus.

A dynamic environment Condition of at least a single patient on the other hand help in finding newer Non - surgical methods to treat Keratoconus.

Exclusion criteria: Some of the exclusion criteria for the study can be unprogressive Keratoconus and old age patient.

Sample size: Three 25 year old male patients with progressive Keratoconus of varied professions and at least a patient with a dynamic environment of work and a 17 year old male patient who is student by profession.

Materials: RGP Contact lenses, Soft K, PROSE contact lenses, Slit Lamp, Surgical instrument if required according to phase of the study.

\section{Bibliography}

1. Sare Ortiz Toquero OD (EC) MsC.

2. Raul Martin OD (EC) MsC, PhD.

3. Gomes JA, Tan D, Ropuano CJ.

4. Siderordi H, Labiris G, Georgatzoglou K, Ditzel F, Siganos C, Kozoballis V.

5. Gonzalez - Meijome, Jose M O.D, F.I.A.C.L.E

6. Jorge, Jorge O.D; De Almeda, Jose B PhD, F.A.A.O.

7. Parafita, Manuel A, M.sC, MD, PhD.

8. AMR Mounia, Mahmoud Mohamed, Farouk, Marwa Mahmoud Abdullah, Envy Mohamed Mastafa.

\section{Assets from publication with us}

- Prompt Acknowledgement after receiving the article

- Thorough Double blinded peer review

- Rapid Publication

- Issue of Publication Certificate

- High visibility of your Published work

Website: www.actascientific.com/

Submit Article: www.actascientific.com/submission.php

Email us: editor@actascientific.com

Contact us: +919182824667 